\title{
ОЦЕНКА ЭФФЕКТИВНОСТИ МЕТОДОВ РЕШЕНИЯ ЗАДАЧ ОБЕСПЕЧЕНИЯ УСТОЙЧИВОСТИ ФУНКЦИОНИРОВАНИЯ РАСПРЕДЕАЕННЫХ ИНФОРМАЦИОННЫХ СИСТЕМ
}

\author{
Д.О. Есиков аспирант, mcgeen4@gmail.com \\ (Тульский государственный университет, просп. Аенина, 92, г. Тула, 300012, Россия)
}

\begin{abstract}
Для принятия обоснованных решений в части организации процессов хранения и обработки данных для обеспечения устойчивости функционирования распределенных информационных систем предложено применять комплекс разработанных математических моделей оптимизации распределения элементов ПО функциональных задач по узлам сети, распределения информационных ресурсов по центрам хранения и обработки данных, состава технических средств системы хранения и обработки данных, распределения резерва информационных ресурсов по центрам хранения и обработки данных. Показано, что данные задачи относятся к классу задач дискретной оптимизации с булевыми переменными.

Для решения формализованных задач предложено и экспериментально проверено применение метода ветвей и границ и генетических алгоритмов. Для повышения эффективности этого метода рекомендован алгоритм предварительного определения порядка ветвления переменных путем однократного решения приближенным методом задачи, двойственной по отношению к исходной. Проведена экспериментальная проверка эффективности метода ветвей и границ для решения задач обеспечения устойчивости функционирования распределенных информационных систем, в том числе с использованием алгоритма предварительного определения порядка ветвления переменных. Дана оценка влияния исходных данных на общую производительность метода ветвей и границ. Определены наиболее эффективные для решения разработанных задач стратегии ветвления переменных. Предложены варианты основных операторов, а также схемы начальной инициализации исходной популяции генетического алгоритма для решения задач обеспечения устойчивости функционирования распределенных информационных систем.

Для повышения качества получаемого генетическим алгоритмом решения обосновано использование адаптивной схемы репродукции особей и островной схемы организации вычислений. Экспериментально проверена эффективность предложенных генетического и островного генетического алгоритмов и определены параметры генетических алгоритмов, обеспечивающие максимальное качество получаемого решения. Подтверждена возможность управления точностью получаемого решения за счет изменения параметров алгоритма при введении ограничений на время решения. Дана сравнительная оценка метода ветвей и границ и островного генетического алгоритма при решении формализованных задач, определены области их эффективного применения.
\end{abstract}

Ключевые слова: распределенная информачионная система, устойчивость функиионирования, математические модели, метод ветвей и грании, двойственная задача, генетический алгоритм, островной генетический алгоритм, адаптивная схема репродукиии.

Устойчивое функционирование распределенных информационных систем (РИС) является залогом эффективной работы организаций и предприятий в различных сферах экономики. Под устойчивостью функционирования системы понимается ее способность выполнять возложенные функции с заданными показателями качества в условиях воздействия внутренних и внешних дестабилизирующих факторов.

В отличие от надежности под устойчивостью подразумевается обеспечение требуемых значений показателей эффективности системы в условиях возможной ее реконфигурации (изменения ее структуры).

В общем случае дестабилизирующие факторы в РИС делятся по местоположению источника на внешние и внутренние, а по характеру возникновения - на случайные и преднамеренные [1].

Наиболее опасными с точки зрения обеспечения целевых показателей функционирования РИС являются дестабилизирующие факторы, в результате воздействия которых ухудшаются параметры процессов обеспечения информационных запросов пользователей, хранения и обработки информации.
К таким факторам можно отнести следующие:

- структурная деградация сети (выход из строя средств обработки информации в узлах распределенной системы, каналов передачи информации);

- разрушение элементов ПО (разрушение данных и кодов исполняемых программ, внесение тонких, труднообнаруживаемых изменений в информационные массивы);

- внедрение программных закладок в другие программы и подпрограммы (вирусный механизм воздействий);

- искажение или уничтожение собственной информации сервера и тем самым нарушение работы сети;

- нарушение целостности (преднамеренное или случайное искажение, разрушение) элементов информационного обеспечения (баз и банков данных), модификация пакетов сообщений и др.

Воздействие этих факторов может иметь катастрофические последствия вплоть до выхода из строя технического, программного и информационного обеспечения систем и, как следствие, привести к нарушениям в процессах обработки инфор- 
мации или к отказам функционирования систем в целом [1]. Для нейтрализации последствий воздействия дестабилизирующих факторов может потребоваться реструктуризация системы с перераспределением информационных ресурсов.

В качестве показателя устойчивости функционирования системы в условиях воздействия $i$-го дестабилизирующего фактора обычно понимается коэффициент устойчивости $K_{\text {учi }}$, определяемый из выражения $K_{\text {учi }}=1-\frac{\left|K_{\text {тр }}-K_{\text {дфi }}\right|}{K_{\text {тр }}}$, где $K_{\text {тр }}-$ требуемое значение показателя качества функционирования системы; $K_{\text {дфi }}$ - значение показателя качества функционирования системы в условиях воздействия $i$-го дестабилизирующего фактора $[2,3]$.

Тогда общий показатель устойчивости системы, выражаемый как коэффициент устойчиво-

сти $K_{\mathrm{y}}$, определяется выражением $K_{\mathrm{y}}=\prod_{i=1}^{l} K_{\text {учi }}$, где I - количество учитываемых дестабилизирующих факторов в системе.

Кроме того, за показатели качества функционирования системы, характеризующие ее устойчивость, могут быть взяты время выполнения рассматриваемого процесса в системе, коэффициент готовности системы, коэффициент эффективности системы и другие [3].

В настоящее время наиболее эффективными для обеспечения устойчивости функционирования РИС являются следующие подходы:

- совершенствование процессов хранения $u$ обработки информации (ПХОИ), в том числе в части обеспечения их устойчивости;

- создание высоконадежных подсистем хранения и обработки данных с учетом их резервирования [4].

Для повышения устойчивости ПХОИ на этапах проектирования и эксплуатации РИС целесообразно выполнять рациональное распределение элементов ПО и информационных массивов по узлам вычислительной сети, а при воздействии дестабилизирующих факторов, в случае структурной деградации сети - их перераспределение [4].

Основу РИС составляет система хранения и обработки данных (СХОД), предназначенная для организации надежного, а также отказоустойчивого хранения данных, высокопроизводительного доступа серверов к устройствам хранения и обработки информации. Обеспечение сохранности информации в СХОД производится путем применения специальных мер организации хранения, восстановления (регенерации) информации, специальных устройств резервирования. Качество обеспечения сохранности информации зависит от еe целостности (точности, полноты) и готовности к постоянному использованию [5-7].

Наиболее простые и эффективные способы обеспечения сохранности информации в СХОД - создание резервных хранилищ данных и реализация мероприятий по оперативному и восстановительному резервированию информации [7].

По типу устройств резервирования резервные хранилища информации в СХОД [5] делятся на хранилища на базе HDD/SSD-дисков (RAID-массивы), на магнитных лентах, на базе оптических дисков.

По количеству уровней резервирования резервные хранилища делятся на одноуровневые и многоуровневые.

При большом количестве разновидностей систем и средств высоконадежного хранения данных, а также их конфигураций стоит задача выбора их состава с учетом решаемых СХОД задач.

Решение задач организации ПХОИ позволяет повысить эффективность функционирования системы за счет снижения издержек, связанных с разрушением информации, включая потери от невозможности решения задач и получения недостоверных выходных результатов, за счет уменьшения времени и затрат на восстановление разрушенной или искаженной информации, рационального использования внешней памяти вычислительных средств для размещения данных РИС и т.д.

Для рациональной организации ПХОИ в РИС в структуре СХОД создают ияентры хранения и обработки информации (ЦХОИ), основной задачей которых является реализация эффективных с точки зрения решаемых в системе задач ПХОИ.

Для обеспечения сохранности информации целесообразно в ЦХОИ создавать подсистемы хранения и обработки данных, основу которых составляют технические средства хранения и обработки информации, интегрируемые в ЦХОИ. В качестве элементов ПО рассматривались программные комплексы, программные модули, осуществляющие решение функциональных задач с использованием информационных массивов в РИС.

Вследствие ограниченности материальных средств, выделяемых на создание РИС, хранение и обработка информации в них осуществляются в ограниченном количестве ЦХОИ, создаваемых в соответствующих узлах сети. В этих центрах аккумулируются мощные вычислительные средства и средства хранения данных, обеспечивающие решение функциональных задач как отдельного сегмента сети, так и системы в целом.

Таким образом, для обеспечения устойчивости функционирования РИС разработан комплекс математических моделей оптимизации

- распределения элементов ПО функциональных задач по узлам сети по критерию минимума объема информации, передаваемой по каналам связи при решении всех функциональных задач;

- распределения информационных ресурсов по ЦХОД по критерию минимума общего значения среднего времени восстановления информации в СХОД; 
- состава технических средств СХОД по критерию максимума значения коэффициента готовности системы хранения данных в целом;

- распределения резерва информационных ресурсов по ЦХОД по критерию минимума среднего времени решения всех задач в РИС [8].

Данные задачи относятся к классу задач комбинаторной оптимизации [9], а именно целочисленного дискретного программирования с булевыми переменными $[9,10]$.

В общем случае математические модели обеспечения устойчивости функционирования РИС могут быть следующими [11].

Определить такие значения $x_{j}(j=1,2, \ldots, N)$, что

$$
F(X)=\min \sum_{j=1}^{N} c_{j} x_{j}
$$

при ограничениях

$$
\begin{aligned}
& x_{j}=\{0,1\}, j=1,2, \ldots, N ; \\
& \sum_{j=1}^{N} a_{i j} x_{j} \leq b_{i}, i=1,2, \ldots, M,
\end{aligned}
$$

где $N$ - число переменных; $M$ - число ограничений.

Для обеспечения эффективности практического применения разработанных математических моделей к методам их решения должны предъявляться определенные требования:

- возможность получения оптимального (квазиоптимального) решения в минимально возможное время;

- возможность управления точностью полученного решения в условиях ограничения на время решения задач;

- невысокая вычислительная сложность;

- возможность распараллеливания процесса получения оптимального (квазиоптимального) решения;

- простота программной реализации вычислительных процедур алгоритма;

- возможность практического применения на вычислительных комплексах с ограниченными характеристиками (невысокой производительности).

Для получения точного решения задачи (1)-(3) существует достаточно большое количество методов дискретной оптимизации $[9,10]$, наиболее распространенным и наиболее широко используемым из которых является метод ветвей и границ [10].

Основными процедурами, составляющими метод ветвей и границ, являются построение дерева возможных вариантов решения, определение оценки границы решения для каждой вершины дерева и отсечение бесперспективных вершин, которые не могут привести к улучшению рекорда [10].

Общая эффективность метода ветвей и границ при решении задач обеспечения устойчивости функционирования РИС непосредственно зависит от выбора стратегии ветвления переменных и способа оценки границ решения, а также от способа организации компьютерных вычислений $[10,12]$. Результаты этого выбора зависят от конкретных условий решения данных задач оптимизации.
Для формирования дерева вариантов в ходе решения задачи наиболее широко используют такие стратегии ветвления переменных [12], как фланговая (левофланговая, правофланговая), фронтальная, локально-избирательная и глобально-поисковая.

Выбор стратегии ветвления оказывает влияние на требуемый для хранения дерева вариантов при решении задачи объем оперативной памяти вычислительного комплекса, а также (за счет особенностей реализации поисковых процедур на дереве решений) на время решения.

Эффективность вычислительных алгоритмов зависит от точности и простоты способа определения верхней или нижней границы возможных решений $[10,12]$. Точность определения верхней (нижней) границы целевых решений напрямую влияет на число бесперспективных вариантов, которые отсеиваются в процессе решения. Увеличение точности расчета границ решений связано с ростом вычислительной сложности алгоритмов и, как следствие, - объема вычислений.

В качестве методов оценки границ решения используют точные методы (симплекс-метод и его модификации) и приближенные методы решения двойственной задачи.

Применение теории двойственности при решении задач методом ветвей и границ позволяет использовать для оценки границы решения приближенные алгоритмы [10]. Однако этот способ оценки границы решения эффективен только при простом и достаточно точном алгоритме решения двойственной задачи.

При решении задачи (1)-(3) методом ветвей и границ условие целочисленности переменных (2) можно заменить условием

$0 \leq x_{j} \leq 1$.

Двойственной задачей по отношению к (1), (2), (4) является задача

$$
Z=\min \left(\sum_{i=1}^{M} b_{i} y_{i}+\sum_{i=M+1}^{M+N} y_{i}\right)
$$

при ограничениях

$$
\begin{aligned}
& \sum_{i=1}^{M} a_{i j} y_{i}+y_{M+j} \geq c_{j}, j=1,2, \ldots, N, \\
& y_{i} \geq 0, i=1,2, \ldots, M+N .
\end{aligned}
$$

В работе [6] предложен приближенный алгоритм оценки границ решения в методе ветвей и границ, основанный на градиентной процедуре решения задачи, двойственной по отношению к исходной. В данном алгоритме использование свойств двойственной задачи и особенностей реализации градиентной процедуры позволяет в процессе решения осуществлять оценку вклада каждой переменной в результат и за счет этого определять порядок ветвления переменных. Для повышения эффективности метода ветвей и границ предложено однократное решение задачи, двойственной по отношению к исходной, с применением данного ал- 
горитма для определения порядка ветвления переменных $[10,13]$.

Экспериментальная оценка эффективности методов решения задач обеспечения устойчивости функционирования РИС проводилась на вычислительной системе со следующими характеристиками: OC Windows 7 Professional x64 Service Pack 1; процессор Intel Core i5-3450 с тактовой частотой $3.10 \mathrm{Ghz}$; объем оперативной памяти $8 \mathrm{~Gb}$.

При экспериментальной проверке методов и алгоритмов решения задач обеспечения устойчивости функционирования РИС методом ветвей и границ ставились следующие задачи:

- оценка эффективности стратегий ветвления переменных в методе ветвей и границ;

- оценка эффективности применения предложенного способа сокращения времени решения задач методом ветвей и границ на основе применения теории двойственности;

- определение параметров генетического алгоритма (ГА), обеспечивающих наилучшее качество получаемого решения;

- определение влияния исходных данных на эффективность методов;

- определение области эффективного применения методов решения формализованных математических моделей.

Исходные данные для экспериментальной проверки эффективности методов решения разработанных математических моделей формировались следующим образом.

Коэффициенты при переменных в целевой функции и ограничениях генерировались как независимые друг от друга целые случайные числа, равномерно распределенные на интервале $[0,100]$. Правые части ограничений вычислялись по зависимости $b_{i}=\frac{Q}{100} \sum_{j=1}^{N} a_{i j}$.

Коэффициент $Q$ определял жесткость ограничений и выбирался из интервала $[30,90]$ с шагом 20 \%. Для каждой размерности решалось по 10 задач.

В качестве характеристик метода ветвей и границ $[14,15]$ использовались следующие величины: время решения задачи $\left(t_{\min }-\right.$ минимальное, $t_{\max }-$ максимальное, $t_{\text {cp }}$ - среднее); число просмотренных вершин ( $V_{\min }$ - минимальное, $V_{\max }$ - максимальное, $V_{\text {ср }}$-среднее); общее время определения границ решения выбранным методом $\left(T_{\min }-\right.$ минимальное, $T_{\max }-$ максимальное, $T_{\mathrm{cp}}-$ среднее).

Для оценки эффективности метода ветвей и границ при решении разработанных задач использовался разработанный программный комплекс [16].

Результаты экспериментальной оценки эффективности применения метода ветвей и границ для решения задач различных размерностей приведены на рисунках 1-4.

Из представленных данных видно, что наименьшее время решения задач обеспечивают глобально-

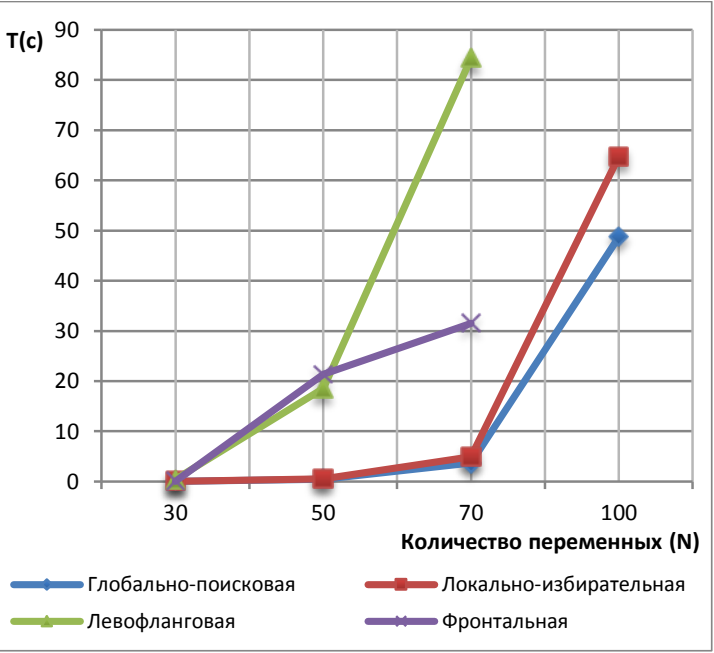

Рис. 1. Зависимость времени решения от размерности задач при количестве ограничений $M=20$ и жесткости $Q=50 \%$

Fig. 1. Dependence of solution time on a problem dimension with the number of restrictions $M=20$, and the rigidity $Q=50 \%$

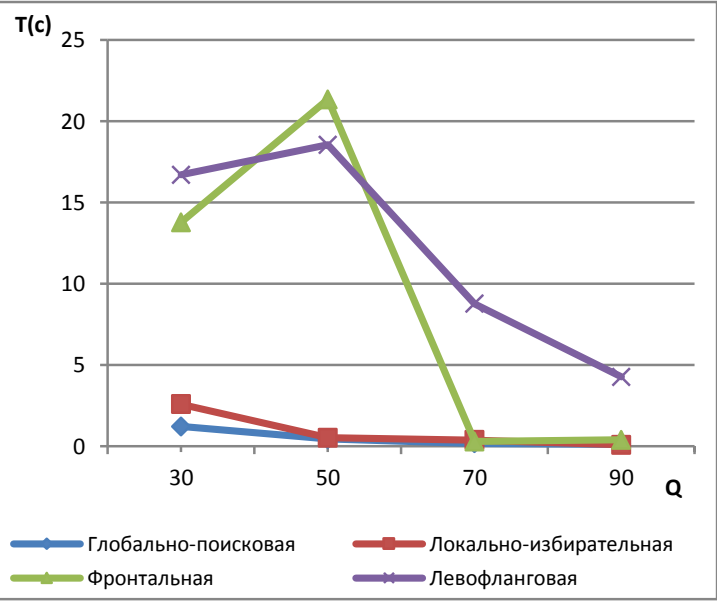

Рис. 2. Зависимость времени решения задач $(50 \times 20)$ от жесткости ограничении $Q$

Fig. 2. Dependence of time for solving problems $(50 \times 20)$ on stiffness of constraints $Q$

поисковая и локально-избирательная стратегии ветвления. Наименее эффективной является фланговая стратегия ветвления. Следует отметить нестабильность поведения фронтальной стратегии ветвления.

При некоторых условиях использование фронтальной стратегии приводит к тому, что процесс решения задачи сводится к полному перебору вариантов решения. Поведение фронтальной стратегии ветвления не зависит от исходных данных решаемых задач и выбора метода оценки границ решения.

Результаты оценки влияния жесткости ограничений на эффективность стратегий ветвления пред- 
Оценка влияния предварительного определения порядка ветвления переменных на эффективность метода ветвей и границ $(Q=0,5)$

Impact assessment of the preliminary determination of the branching order of variables on the efficiency of the branch and bound method $(Q=0,5)$

\begin{tabular}{|c|c|c|c|c|}
\hline \multirow{3}{*}{$\begin{array}{c}\text { Размерность } \\
\text { задачи }\end{array}$} & \multicolumn{4}{|c|}{ Среднее время решения $t$ (сек.) } \\
\hline & \multicolumn{2}{|c|}{ Без определения порядка ветвления } & \multicolumn{2}{|c|}{ С определением порядка ветвления } \\
\hline & Симплекс-метод & Приближенный метод & Симплекс-метод & Приближенный метод \\
\hline $30 \times 10$ & 0,13 & 0,24 & 0,04 & 0,06 \\
\hline $50 \times 10$ & 0,54 & 60,16 & 0,39 & 5,34 \\
\hline $60 \times 10$ & 3,95 & 76,69 & 1,73 & 7,04 \\
\hline $70 \times 10$ & 16,87 & 284 & 2,73 & 8,06 \\
\hline $100 \times 10$ & 367,14 & - & 26,31 & 4002,68 \\
\hline
\end{tabular}

ставлены на рисунках 2 и 3 . На них видно, что со снижением жесткости ограничений число просмотренных вершин и, соответственно, время решения задач уменьшаются. Это влияние особенно сильно проявляется с увеличением размерности задачи (числа переменных). Так, на размерности 100 переменных и 30 ограничений снижение жесткости ограничений с $Q=50 \%$ до $Q=90 \%$ сокращает время решения задач в 29 раз. Это объясняется снижением количества перспективных вершин среди не ответвленных на дереве решений. Таким образом, повышение жесткости ограничений приводит к значительному увеличению числа просматриваемых методом вариантов решения, что ставит под сомнение целесообразность использования в этих случаях таких стратегий ветвления переменных, как фланговая, фронтальная и их модификации.

Анализ результатов расчетов, представленных на рисунке 3 , показывает, что в зависимости от выбранной стратегии ветвления 27-89\% от времени решения задачи составляет совокупное время определения границ решения симплекс-методом.

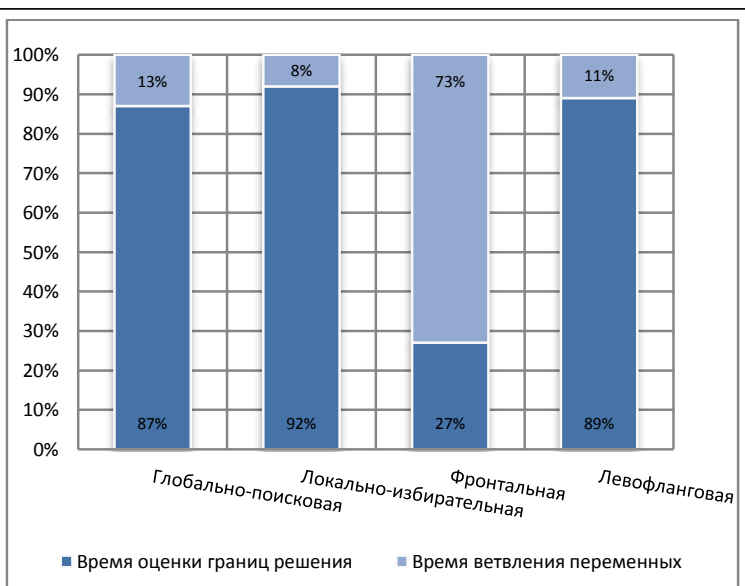

Рис. 3. Доля времени ветвления переменных относительно общего времени решения задачи $(70 \times 10)$

Fig. 3. The variable branch-time share compared with total problem solving time $(70 \times 10)$
При оценке влияния применения алгоритма определения порядка ветвления переменных на эффективность метода ветвей и границ в качестве методов оценки границ решения использовались симплекс-метод и приближенный метод решения двойственной задачи.

Результаты экспериментальной проверки эффективности влияния предварительного определения порядка ветвления переменных приведены в таблице 1 и на рисунке 4 [13-15]. В качестве методов оценки границ решения использовались симплекс-метод и рассмотренный выше приближенный метод решения двойственной задачи на основе применения градиентной процедуры, в качестве стратегии ветвления переменных - глобально-поисковая стратегия.

Из таблицы 1 и рисунка 4 видно, что предварительное определение порядка ветвления переменных при решении задач различных размерностей позволяет снизить время решения задач в 3-14 раз. Результаты решения стабильны независимо от способа оценки верхней границы. Это говорит об эф-

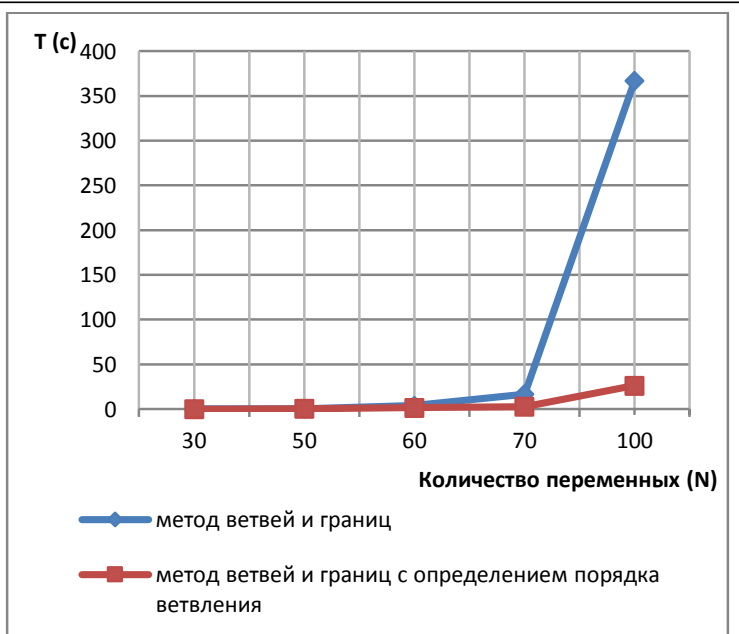

Рис. 4. Зависимость времени решения задач $(m=10)$ от количества переменных в цуелевой функичи

Fig. 4. The problem solving time $(m=10)$ depending on the number of variables in the objective function 
фективности предложенного алгоритма и целесообразности его использования при решении практических задач методом ветвей и границ.

Таким образом, экспериментальная проверка эффективности метода ветвей и границ показала, что наиболее эффективными при решении задач обеспечения устойчивости РИС являются глобально-поисковая и локально-избирательная стратегии ветвления переменных. Увеличение жесткости ограничений приводит к значительному росту времени решения задач методом ветвей и границ независимо от используемых стратегий ветвления и методов оценки границ решения. Применение предложенного способа ранжирования переменных по уровню влияния на результат решения задачи для организации процесса ветвления переменных совместно с точным методом оценки границ решения обеспечивает существенное (от 3 до 14 раз) повышение производительности метода ветвей и границ.

Достоинством метода ветвей и границ является точность получаемого решения за ограниченное время, недостатком - экспоненциальная зависимость времени решения от размерности решаемых задач [15].

Для практического применения формализованных задач обеспечения устойчивости функционирования РИС решения задачи в условиях жестких ограничений на время решения предложено и экспериментально проверено использование ГА как варианта стохастического эволюционного поиска решения. Основу ГА составляет хромосома (особь) $[17,18]$.

Применительно к данной задаче хромосома (особь) состоит из набора $N$ генов, каждый из которых представляет собой значение соответствующей переменной решаемой задачи и принимает значение 0 или 1.

В ГА над хромосомами выполняются следующие действия: определение значения функции приспособленности, скрещивание (кроссинговер), мутация, отбор особей для скрещивания.

Значение функции приспособленности используется для оценки качества варианта решения, соответствующего значениям генов хромосомы.

Функция приспособленности рассчитывается как значение целевой функции.

Если геном хромосомы соответствует недопустимому значению переменных (не выполняются ограничения), функция приспособленности возвращает значение $<0$ (например -100 000), в противном случае - положительное число, значение $W$.

Для генерации новых вариантов решения (особей) в ГА используют механизмы отбора особей, скрещивания и мутации хромосом.

Для работы ГА [17-19] обычно используют следующие популяции:

- исходная популяция; служит для хранения особей, участвующих в текущем шаге ГА; на начальном шаге ГА исходная популяция обычно генерируется случайным образом;

- родительская популяция (родительский пул); в родительскую популяцию отбираются все особи исходной популяции, значения функции приспособленности которых не меньше среднего значения функции приспособленности для исходной популяции в целом;

- элитная популяция; служит для сохранения лучших особей родительской (исходной) популяции;

- дочерняя популяция; формируется путем многократного выполнения операций отбора особей, кроссинговера, мутации; на основе дочерней популяции формируется исходная популяция для следующего шага ГА.

Для упрощения процедур ГА предлагается использовать исходную популяцию постоянного размера. В случае, если на какой-либо итерации вновь сформированная популяция, которая на следующей итерации будет использоваться как исходная, имеет размер, менее заданного, предлагается дополнять ее до требуемого размера случайно сгенерированными особями.

Особи для скрещивания выбираются из родительской популяции. Первая хромосома выбирается случайным образом.

Выбор второй особи для скрещивания возможен по следующим вариантам:

- случайный выбор; вторая особь выбирается случайно из родительской популяции; данный вариант наиболее прост для реализации, однако вследствие своей природы не может гарантировать качество получаемого решения;

- родственное скрещивание на основе анализа расстояния между особями; в качестве расстояния между хромосомами для решаемой задачи целесообразно использовать расстояние Хэмминга, определяемое для двух особей как $S=\sum_{i=1}^{N}\left|g_{i}^{1}-g_{i}^{2}\right|$, где $g^{1}{ }_{i}$ и $g^{2}{ }_{i}-$ значения $i$-х генов 1-й и 2-й особей.

В соответствии с этим различают близкородственное (инбридинг) и дальнеродственное (аутбридинг) скрещивание.

Аутбридинг - выбирается хромосома с максимальным значением расстояния Хэмминга до первой выбранной: $S \rightarrow \max$. Данный вид отбора обеспечивает снижение скорости сходимости алгоритма к локальному экстремуму и увеличивает вероятность достижения глобального экстремума.

Инбридинг - выбирается хромосома с минимальным расстоянием от выбранной первой: $S \rightarrow \min$. Данный вид отбора обеспечивает максимально быструю сходимость алгоритма к локальному экстремуму.

Для обеспечения сохранения лучших найденных особей содержимое элитной популяции автоматически включается в дочернюю популяцию на текущем шаге алгоритма. 
Размер элитной популяции $L^{e l}$ определяется как $L^{e l}=k^{e l} L^{s r}$, где $k^{e l}$ - коэффициент элитизма; $L^{s r}-$ размер исходной популяции.

Операция скрещивания (кроссинговер) выполняется над парой хромосом, являющихся родительскими. Результат операции - две хромосомы-потомка.

Исследования показали, что одним из наиболее эффективных с точки зрения конечного результата является универсальный кроссинговер, который выполняется следующим образом.

Генерируется шаблон двоичных чисел длиной $N$. Шаблон указывает, какие гены должны наследоваться от первого родителя (остальные гены берутся от второго родителя).

Для первого потомка: если в $j$-й позиции шаблона 1 , то $j$-й ген для данного потомка берется от первого родителя, если 0 - от второго. Для второго потомка: если в $j$-й позиции шаблона 1 , то $j$-й ген для данного потомка берется от второго родителя, а если 0 - от первого.

Кроме того, потенциально хороший результат может показать триадный кроссинговер с формированием маски кроссинговера из лучшей особи элитной популяции. Он аналогичен универсальному, за исключением того, что маска кроссинговера формируется не случайно, а соответствует лучшей особи в элитной популяции.

В качестве претендента на использование возможно применение триадного кроссинговера на основе анализа схемы [20] в следующем виде.

Пусть $g^{1}, g^{2}$ и $g^{3}$ - родительские хромосомы; $f_{g^{1}}, f_{g^{2}}, f_{g^{3}}-$ значения функции приспособленности родительских особей. $g^{1}$ и $g^{2}$ выбираются в соответствии с текущей схемой отбора особей, $g^{3}-$ случайным образом из особей родительской популяции.

Так как гены принимают только значения из набора $\{0,1\}$, обозначим через $P_{0}(j)$ и $P_{1}(j)$ частоты появления значений 0 и 1 для $j$-го гена в родительской популяции соответственно. Тогда для всех $j$, $j=1,2, \ldots, N$, определим значения генов дочерних особей $\bar{g}_{j}^{1}$ и $\bar{g}_{j}^{2}$ по следующим правилам.

Если $g_{j}^{1}=g_{j}^{2}$, то $\bar{g}_{j}^{1}=g_{j}^{1}$.

Если $g_{j}^{1} \neq g_{j}^{2}$, то

при $g_{j}^{1}=0$, если $f_{g^{1}} P_{0}(j) \geq f_{g^{2}} P_{1}(j), \bar{g}_{j}^{1}=g_{j}^{1}$ и

$\bar{g}_{j}^{1}=g_{j}^{2}$ в противном случае;

при $g_{j}^{1}=1$, если $f_{g^{1}} P_{1}(j) \geq f_{g^{2}} P_{0}(j), \bar{g}_{j}^{1}=g_{j}^{1}$ и

$\bar{g}_{j}^{1}=g_{j}^{2}$ в противном случае.

Если $f_{g^{1}}>f_{g^{2}}$, то $g^{4}=g^{1}, f_{g^{4}}=f_{g^{1}}$ и $g^{4}=g^{2}$, $f_{g^{4}}=f_{g^{2}}$ в противном случае.

Если $g_{j}^{4}=g_{j}^{3}$, то $\bar{g}_{j}^{2}=g_{j}^{4}$.

Если $g_{j}^{4} \neq g_{j}^{3}$, то при $g_{j}^{4}=0$, если $f_{g^{4}} P_{0}(j) \geq f_{g^{3}} P_{1}(j), \bar{g}_{j}^{2}=g_{j}^{4}$ и $\bar{g}_{j}^{2}=g_{j}^{3}$ в противном случае;

при $x_{j}^{4}=1$, если $f_{g^{4}} P_{1}(j) \geq f_{g^{3}} P_{0}(j), \bar{g}_{j}^{2}=g_{j}^{4}$ и $\bar{g}_{j}^{2}=g_{j}^{3}$ в противном случае.

Нетрудно заметить, что при $P_{1}(j)=1$ или $P_{0}(j)=1$ все особи в популяции имеют одинаковое значение $j$-го гена и это значение гарантированно перейдет по наследству потомкам.

Операция мутации выполняется над выбранной хромосомой. Данный оператор необходим для «выбивания» популяции из локального экстремума и препятствует преждевременной сходимости. Это достигается за счет того, что изменяется случайно выбранный ген $g_{i}(i=1,2, \ldots, N)$ в хромосоме. Значение выбранного гена (переменной исходной задачи в данном варианте решения) инвертируется.

Введем обозначения: $N^{g e n}-$ число поколений, формируемых островом в одной итерации ГА; $P_{c}-$ вероятность выполнения скрещивания хромосом; $P_{m}-$ вероятность мутации хромосомы.

Простой ГА состоит из следующих шагов.

1. Начальная инициализация исходной популяции случайно сформированными хромосомами.

2. $k=0$.

3. Определение среднего значения функции принадлежности хромосом исходной популяции.

4. Формирование родительской популяции из особей исходной популяции, значение функции принадлежности которых не меньше среднего значения функции принадлежности в исходной популяции. Определение длины родительской популяции $L^{p r}$

5. Формирование элитной популяции из $L^{e l}$ лучших особей исходной популяции.

6. Включение в дочернюю популяцию хромосом, входящих в элитную популяцию.

7. $i=0$.

8. Случайный выбор хромосомы $g_{1}$ из родительской популяции.

9. В соответствии с заданным способом выбора второй хромосомы для скрещивания выбор из родительской популяции хромосомы $g_{2}$.

10. Формирование случайного числа rnd $\epsilon$ $\in[0,1]$. Если $r n d \leq P_{c}$, выполнение процедуры скрещивания хромосом $g_{1}$ и $g_{2}$ с получением новых хромосом $\bar{g}_{1}$ и $\bar{g}_{2}$, для которых выполняется расчет значения функции принадлежности. Если $r n d>P_{c}$, присвоение $\bar{g}_{1}$ значения $g_{1}$ и $\bar{g}_{2}$ значения $g_{2}$.

11. Формирование случайных чисел из интервала $[0,1] r n d_{1}$ и $r n d_{2}$. Если $r n d_{1} \leq P_{m}$, выполнение операции мутации с хромосомой $\bar{g}_{1}$. Если $r n d_{2} \leq P_{m}$, выполнение операции мутации с хромосомой $\bar{g}_{2}$.

12. Включение с учетом результатов проверки на совпадение хромосом и допустимость значения функции принадлежности хромосом $\bar{g}_{1}$ и $\bar{g}_{2}$ в дочернюю популяцию. 
13. $i=i+1$.

14. Если $i<L^{p r} / 2$, переход к шагу 8 , в противном случае - к шагу 15.

15. Дополнение дочерней популяции хромосомами из родительской популяции, не входящими в состав дочерней. Определение размера дочерней популяции $L^{\text {ch }}$

16 . Если $L^{c h}<L^{s r}$, дополнение дочерней популяции случайно сформированными хромосомами в количестве $L^{s r}-L^{c h}$.

17. Очистка исходной, родительской и элитной популяций. Перенос хромосом из дочерней популяции в исходную. Очистка дочерней популяции.

18. $k=k+1$.

19. Если $k<N^{g e n}$, переход к шагу 3 , иначе - к шагу 20.

20. Формирование элитной популяции.

21. Конец. Результат - лучшая особь в элитной популяции.

При решении задач обеспечения устойчивости функционирования РИС рассматривались две схемы генерации особей для формирования исходных популяций:

- случайная генерация варианта решения (особи) с последующей проверкой на выполнимость ограничений;

- последовательная генерация варианта решения (особи) с поэтапной проверкой выполнимости ограничений.

Обе схемы основаны на случайной генерации значений переменных при решении задач обеспечения устойчивости функционирования РИС.

Первая схема потенциально имеет больше шансов сформировать оптимальное решение уже в начальной популяции, однако требует существенного отсева вариантов, не удовлетворяющих ограничениям.

В соответствии со второй схемой, если на последующем этапе полученные значения переменных в варианте решения задач обеспечения устойчивости функционирования РИС не удовлетворяют ограничениям, за вариант решения берется особь, полученная на предыдущем этапе. Данная схема формирует исходную популяцию за количество шагов, незначительно превышающее размер популяции.

Экспериментальная проверка показала, что реализация первой схемы занимает до 30 \% от общего времени решения задачи. Причем с ростом размерности задачи, количества ограничений, жесткости ограничений эта доля времени существенно возрастает.

Применение второй схемы позволяет сократить долю времени на формирование исходной популяции до 1-2 \% от общего времени решения. Причем при ужесточении или росте числа ограничений данная величина растет незначительно.

Таким образом, при решении практических задач целесообразно применять схему последова- тельного формирования вариантов решения (особей) при создании исходной популяции.

Определено, что наилучшие показатели при решении задач обеспечения устойчивости функционирования РИС простой ГА имеет при $P_{c}=0,8$, $P_{m}=0,1, k_{e l}=0,1$.

Выбор схемы репродукции оказывает существенное влияние на качество получаемого результата. Однако эффективность схемы репродукции существенно зависит от результатов начальной инициализации исходной популяции. Неверный выбор схемы репродукции обычно приводит к существенному ухудшению качества получаемого решения. В связи с тем, что инициализация исходной популяции осуществляется случайным образом, заранее осуществить эффективный выбор схемы репродукции не представляется возможным. Поэтому предлагается использовать адаптивную схему репродукции. Для этого в каждую хромосому необходимо включить числовые метки используемых для ее получения схемы отбора и варианта кроссинговера.

На каждом шаге алгоритма (при формировании нового поколения особей) необходимо определить количество особей в родительской популяции, сформированных с использованием каждой схемы отбора и каждого вида кроссинговера, и рассчитать частоту (вероятность) использования схем отбора и вариантов кроссинговера.

При начальной инициализации исходной популяции для всех хромосом данным меткам присваивается значение -1, а частоты применения схем отбора и вариантов кроссинговера принимают равновероятными. При этом схемы отбора особей и варианты кроссинговера используются случайным образом в соответствии с полученными частотами (вероятностями) их применения.

Для реализации адаптивной схемы репродукции рассматривались схемы отбора особей: аутбридинг, инбридинг, случайный отбор, а также варианты кроссинговера: одноточечный, двухточечный, универсальный, триадный (с формированием маски кроссинговера из лучшей особи элитной популяции), триадный с учетом схемы.

В связи с незначительным влиянием мутации особей на качество получаемого решения использовался только один вариант мутации - одноточечная мутация.

Экспериментальная проверка эффективности ГА осуществлялась с применением разработанной программы [21]. Результаты экспериментальной проверки представлены в таблицах 2, 3 и на рисунке 5.

Из таблиц 2 и 3 видно, что качество получаемого решения при использовании простого ГА вследствие сходимости к случайному локальному экстремуму может существенно варьироваться в зависимости от результата начальной инициализации исходной популяции [22]. 


Время формирования 1-го поколения ГА $\begin{gathered}\text { Таблица } \\
\text { (сек.) } \\
\text { Table } 2\end{gathered}$
Time of formation of the 1st generation of GA (sec.)
\begin{tabular}{|c|c|c|c|c|}
\hline \multirow{2}{*}{$\begin{array}{c}\text { Размер- } \\
\text { ность } \\
\text { задачи }\end{array}$} & $\begin{array}{c}\text { Аутбри- } \\
\text { динг }\end{array}$ & $\begin{array}{c}\text { Инбри- } \\
\text { динг }\end{array}$ & $\begin{array}{c}\text { Случай- } \\
\text { ный }\end{array}$ & $\begin{array}{c}\text { Адаптив- } \\
\text { ный }\end{array}$ \\
\hline $70 \times 4$ & 0,00207 & 0,00205 & 0,00207 & 0,00208 \\
\hline $100 \times 4$ & 0,00376 & 0,00376 & 0,00371 & 0,00375 \\
\hline $150 \times 4$ & 0,008745 & 0,008761 & 0,008381 & 0,008636 \\
\hline
\end{tabular}

Экспериментальная проверка показала, что простой ГА с высокой долей вероятности позволяет получить оптимальное решение для задач малой (до 70 переменных) размерности. В случае получения в качестве решения локального экстремума отклонение от оптимума при том же времени решения составляет до 1,5\%.

На рисунке 5 представлена зависимость времени формирования одного поколения особей в ГА от размерности (числа переменных) решаемой задачи.

Из таблицы 2 и рисунка 5 видно, что с ростом числа переменных решаемой задачи время формирования одного поколения ГА растет экспоненциально. Данное обстоятельство позволяет судить о том, что время решения задач ГА будет нелинейно расти с ростом размерности задач. Степень нелинейности зависит от выбранных параметров алгоритма. Кроме того, с ростом размера популяции время решения задачи растет в квадратичной зависимости. Качество получаемого решения при использовании простого ГА вследствие сходимости к случайному локальному экстремуму может существенно варьироваться в зависимости от результата начальной инициализации исходной популяции $[17,22]$.

Кроме того, из таблицы 3 видно, что с ростом размерности задачи выше 100 переменных область поиска решения столь существенно расширяется,

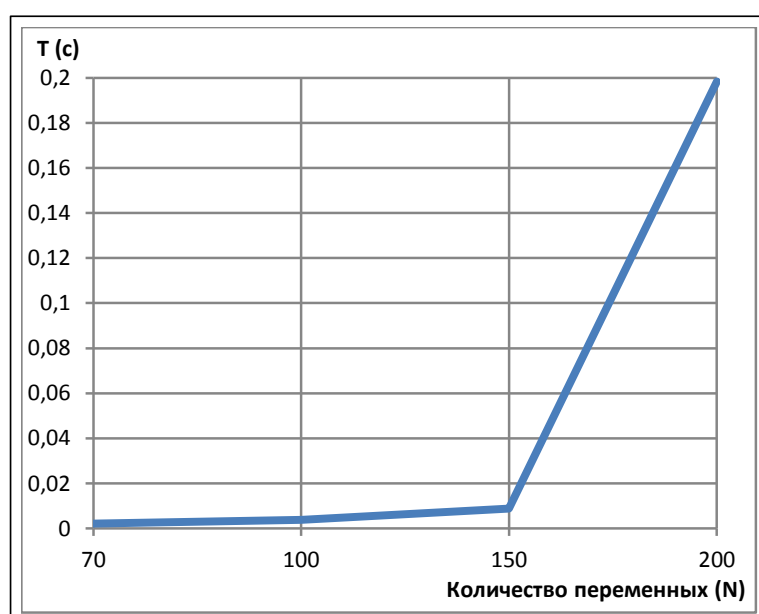

Рис. 5. Зависимость времени формирования 1-го поколения от размерности задачи (случайный отбор)

Fig. 5. Dependence of the 1 st generation formation time on a problem dimension (random selection)

что вероятность получения оптимального решения простым ГА значительно снижается независимо от выбора параметров алгоритма.

Наилучшие схемы репродукции - случайный отбор и адаптивная (обеспечивают наискорейшее достижение оптимального решения при минимальном количестве итераций). При этом эффективность адаптивной схемы растет по сравнению со случайной с ростом размерности задачи. Это объясняется несколько большим разнообразием особей в исходной популяции, получаемым при использовании адаптивной схемы. Данная схема показывает более стабильный по качеству получаемого решения результат. При этом время решения незначительно выше, чем при использовании случайной схемы.

В связи с тем, что с ростом размерности решаемых задач простой ГА в большинстве случаев получает в качестве решения локальный экстремум, значение и близость которого к оптимальному существенно зависят от результатов начальной ини-

Таблица 3

Результаты экспериментальной проверки эффективности простого ГА для решения задачи обеспечения устойчивости функционирования РИС

Table 3

Results of experimental verification of the simple GA effectiveness to solve the task of ensuring the sustainability of distributed information systems

\begin{tabular}{|c|c|c|c|c|c|c|c|c|}
\hline \multirow[b]{2}{*}{ 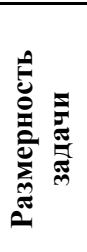 } & \multicolumn{2}{|c|}{ Аутбридинг } & \multicolumn{2}{|c|}{ Инбридинг } & \multicolumn{2}{|c|}{ Случайный } & \multicolumn{2}{|c|}{ Адаптивный } \\
\hline & 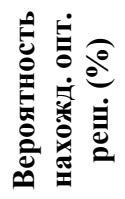 & 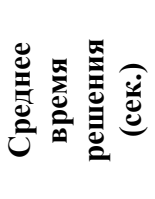 & 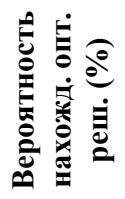 & 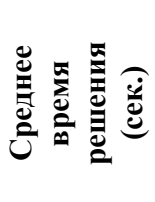 & 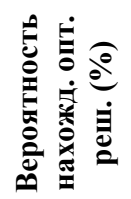 & 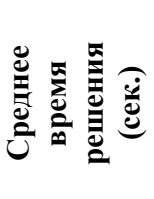 & 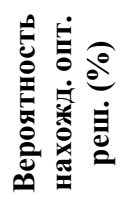 & 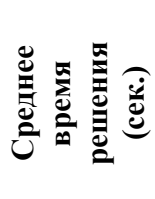 \\
\hline $70 \times 4$ & 0,2 & 1,982 & 0,4 & 1,971 & 1,0 & 1,983 & 0,8 & 2,001 \\
\hline $100 \times 4$ & 0 & 12,032 & 0 & 12,02 & 0,2 & 11,861 & 0,2 & 11,983 \\
\hline $150 \times 4$ & 0 & 69,96 & 0,1 & 70,088 & 0 & 67,048 & 0 & 69,09 \\
\hline
\end{tabular}


циализации исходной популяции, для получания более стабильного результата предлагается использовать островной ГА (ОГА), являющийся модификацией простого ГА [23].

Сущность ОГА заключается в следующем. Одновременно и независимо друг от друга (параллельно) работают несколько простых ГА со своими популяциями. Каждый из них вследствие случайной начальной инициализации исходной популяции сходится к своему локальному экстремуму (острову). С определенной периодичностью (через определенное число поколений) по определенной схеме ГА обмениваются заданным числом лучших особей (осуществляется миграция особей), после чего процесс решения продолжается. Совокупность шагов алгоритма, завершающаяся обменом лучшими особями между островами (миграцией особей), составляет одну итерацию ОГА. Подобная схема позволяет обеспечить выход простых ГА в каждом из островов из области локального экстремума, обеспечить большее разнообразие особей в популяциях и с большей вероятностью получить лучшее решение (в том числе достичь глобального экстремума). В качестве схемы миграции особей чаще всего используются миграция с топологией полной сети, миграция с топологией кольца и их модификации [22, 23].

Введем обозначения: $N^{\text {Lnd }}-$ число островов; $N^{\text {Step }}$ - число итераций ОГА.

ОГА с миграцией особей с топологией кольца состоит из следующих шагов.

1. Для каждого $k$-го острова $\left(k=1,2, \ldots, N^{\text {Lnd }}\right)$ выполнить инициализацию исходной популяции путем заполнения ее особями, сгенерированными случайным образом (в исходную популяцию не включаются нежизнеспособные особи, повторное включение уже имеющейся особи не допускается) и формирование элитной популяции объемом $L^{e l}$ особей.

2. $i=0$.

3. Для каждого острова $\left(k=1,2, \ldots, N^{\text {Lnd }}\right)$ осуществляется последовательная эволюция поколений в количестве $N^{\text {gen }}$ (выполнение простого ГА с заданной схемой репродукции).

4. Если $N^{\text {Lnd }}>1$, для каждого $k$-го острова $\left(k=1,2, \ldots, N^{L n d}-1\right)$ осуществляется перенос $m$ $\left(m=L^{e l} / 2\right)$ лучших особей из элитной популяции в исходную популяцию $j$-го $(j=k+1)$ острова путем замещения худших особей. Для случая $k=N^{L n d}$ осуществляется перенос лучших особей в 1-й остров.

5. $i=i+1$.

6. Если $i<N^{\text {Step }}$, перейти к п. 3. В противном случае к п. 7.

7. Сформировать результирующую популяцию из особей, составляющих элитные популяции каждого из островов.

8. Из результирующей популяции выбрать лучшую особь - результат решения.

9. Конец.
Помимо адаптивной схемы репродукции, возможно использование ее упрощенного варианта смешанной или гибридной схемы, при которой вариант селекции и кроссинговера для каждого острова выбирается при инициализации случайно и однократно.

Результаты экспериментальной проверки эффективности ОГА для решения задач дискретного линейного целочисленного программирования с булевыми переменными представлены в таблицах 4, 5 и на рисунках 6-8.

В таблице 4 показаны экспериментально полученные параметры ОГА при решении задач обеспечения устойчивости функционирования РИС различной размерности, обеспечивающие максимально возможные шансы получения оптимального решения [22]. Из таблицы видно, что для получения решения максимального качества в ОГА количество островов должно быть не меньше $0,025 N$, количество особей в популяции $-0,6 N$, количество поколений в итерации должно быть в интервале от $0,9 N$ до $1,2 N$, число итераций - не меньше $0,05 N$.

В таблице 5 представлены результаты решения задач различной размерности ОГА с применением параметров алгоритма, приведенных в таблице 2 . Из таблицы 5 видно, что ОГА с достаточно высокой степенью вероятности (по сравнению с простым ГА) получает оптимальное решение задач, в том числе большой размерности (число переменных более 150). В случае получения локального экстремума отклонение от оптимального решения не превышало $0,2 \%$.

На рисунке 6 показана зависимость значения целевой функции решаемой задачи (размерность $150 \times 4)$ от числа итераций. ОГА осуществляет последовательное приближение к глобальному экстремуму. При этом с 4-й итерации скорость прироста качества получаемого решения существенно снижается. Это свидетельствует о том, что в случае

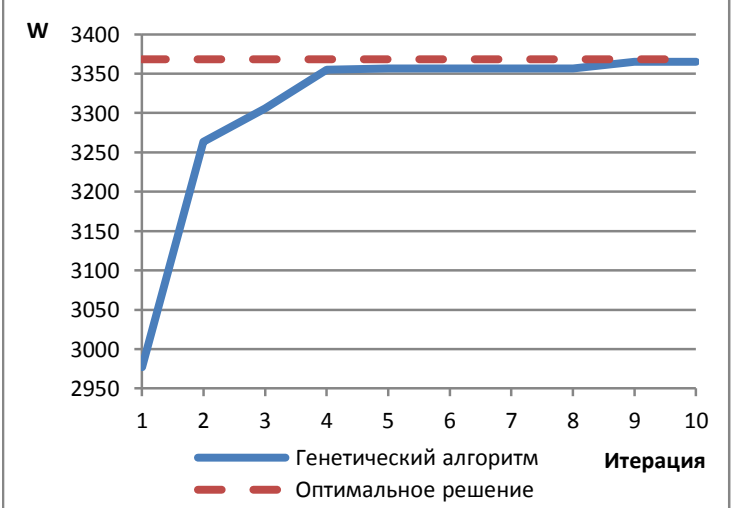

Рис. 6. Зависимость значения целевой функции от итерациии (задача 150×4)

Fig. 6. Dependence of the objective function on the iteration (task $150 \times 4$ ) 
Параметры и характеристики ОГА для решения задач дискретного целочисленного

Таблица 4 программирования с булевыми переменными

Parameters and characteristics of an island genetic

Table 4 algorithm for solving the problems of discrete integer programming with boolean variables

\begin{tabular}{|c|c|c|c|c|c|}
\hline \multirow{2}{*}{$\begin{array}{c}\text { Параметры } \\
\text { и характеристики }\end{array}$} & \multicolumn{4}{|c|}{ Количество переменных N } & \multirow{2}{*}{$\begin{array}{c}\text { Значение } \\
\text { параметра }\end{array}$} \\
\hline & 70 & 100 & 150 & 200 & \\
\hline Количество островов & 4 & 4 & 5 & 8 & $0,025 N$ \\
\hline Количество итераций & 6 & 4 & 8 & 10 & $0,05 N$ \\
\hline Количество особей в популяции & 50 & 60 & 80 & 120 & $0,6 N$ \\
\hline Длина периода миграции особей (поколений) & - & 120 & 160 & 180 & $0,9 N \div 1,2 N$ \\
\hline Поколений в итерации & 40 & 200 & 200 & 200 & - \\
\hline Среднее время решения (сек.) & 2,0 & 12,1 & 68,8 & 396,9 & - \\
\hline Среднее время решения задач методом ветвей и граний & 0,2 & 13,99 & 74,05 & 1279,58 & - \\
\hline
\end{tabular}

Таблица 5

Результаты экспериментальной проверки эффективности ОГА для решения задачи обеспечения устойчивости функционирования РИС

Results of experimental verification of the effectiveness of the island GA for solving the problem of ensuring the sustainability of distributed information systems

\begin{tabular}{|c|c|c|c|c|c|c|c|c|}
\hline \multirow[b]{2}{*}{ 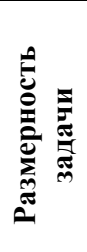 } & \multicolumn{2}{|c|}{ Аутбридинг } & \multicolumn{2}{|c|}{ Инбридинг } & \multicolumn{2}{|c|}{ Случайный } & \multicolumn{2}{|c|}{ Адаптивный } \\
\hline & 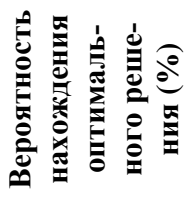 & 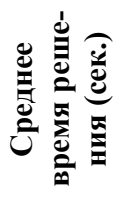 & 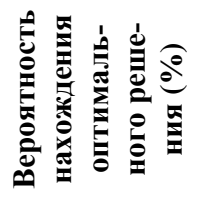 & 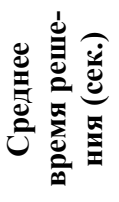 & 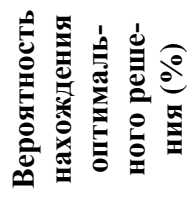 & 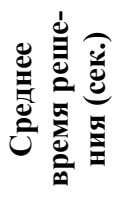 & 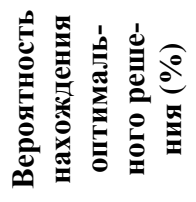 & 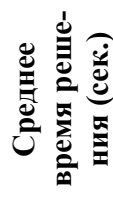 \\
\hline $70 \times 4$ & 0,70 & 2,007 & 0,50 & 2,00 & 0,90 & 2,00 & 0,90 & 2,031 \\
\hline $100 \times 4$ & 0,00 & 12,19 & 0,30 & 12,18 & 0,90 & 11,91 & 0,75 & 11,98 \\
\hline $150 \times 4$ & 0,10 & 68,82 & 0,10 & 68,79 & 0,70 & 66,38 & 0,70 & 70,13 \\
\hline
\end{tabular}

жесткого лимита времени, отводимого на решение задачи, когда можно удовлетвориться рациональным (квазиоптимальным) решением, достаточно использования 4-5 итераций алгоритма для данной размерности задач, что позволит сократить время поиска решения не менее чем на 40-60 \%.

Время решения задачи ОГА линейно растет с увеличением числа островов.
На рисунках 7 и 8 представлены результаты работы адаптивной схемы в ОГА при решении задачи размерности $100 \times 4$.

К 5-6-й итерациям процедура адаптации сходится к выбору одной из схем отбора особей или их устойчивой комбинации. Аналогичная ситуация наблюдается в адаптации выбора кроссинговеров к 6-8-й итерациям алгоритма. Это может свидетель-

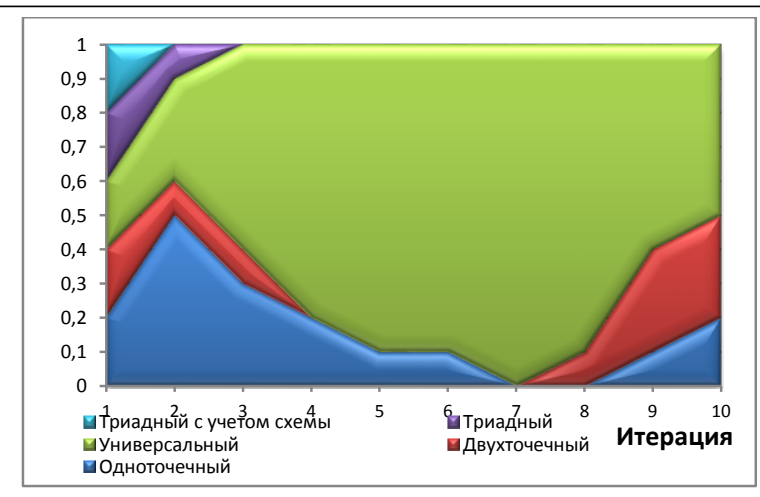

a)

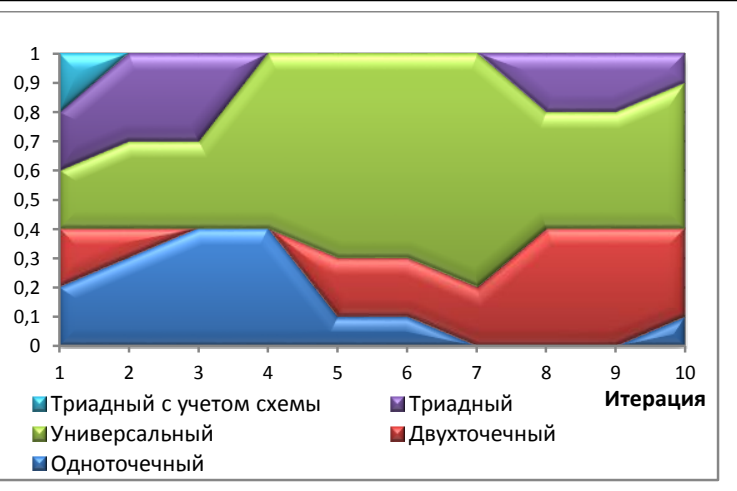

б)

Рис. 7. Результаты экспериментальной проверки адаптации выбора варианта кроссинговера: а) остров 1; б) остров 2

Fig. 7. The results of experimental verification of selection crossingover adaptation options: a) island 1; 6) island 2 


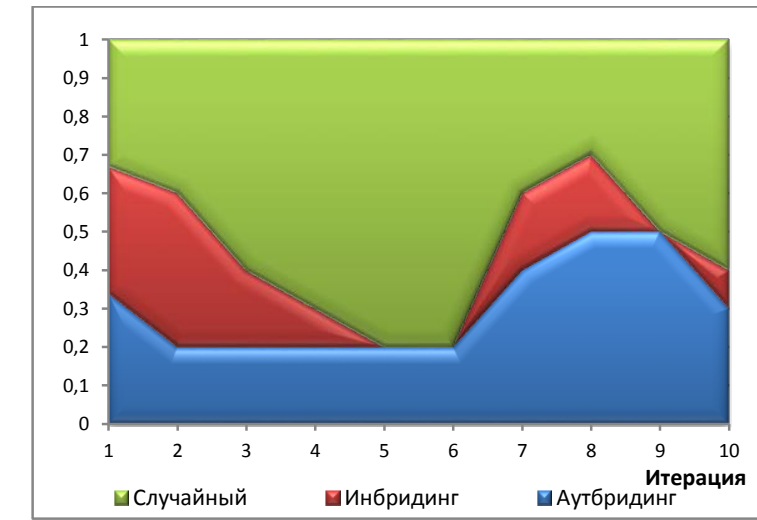

a)

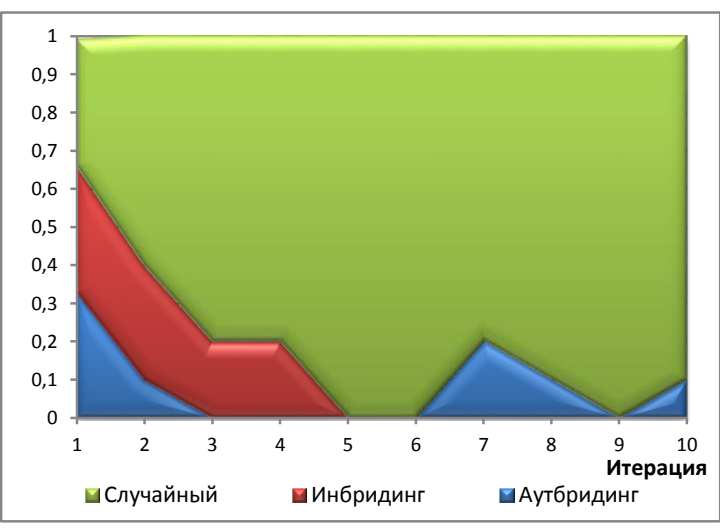

б)

Рис. 8. Результаты экспериментальной проверки адаптации выбора варианта схемы отбора особей: а) остров 1 ; б) остров 2

Fig. 8. The results of the experimental testing of adaptation options select species sampling scheme: a) island 1; 6) island 2

ствовать о том, что ОГА к 7-й итерации осуществляет достаточное и устойчивое сближение к локальному экстремуму в каждом из островов. При этом миграция особей между островами до схождения к локальному экстремуму не оказывает кардинального влияния на работу схемы адаптации и лишь улучшает состав популяции, обеспечивая снижение скорости схождения к ближайшему локальному экстремуму. После 5-7-й итераций (когда острова в значительной степени приблизились к локальным экстремумам) обмен лучшими особями приводит к кратковременному выходу схемы адаптации из равновесного состояния.

Применение триадных кроссинговеров с учетом схемы и с использованием в качестве маски кроссинговера лучшей особи не дают требуемого качества решения; и при использовании адаптивной схемы репродукции, и в большинстве случаев к 3-4-й итерации ОГА отсекаются от применения. При этом количество лучших особей, полученных с применением универсального кроссинговера, в популяциях во всех островах ОГА составляет порядка $46 \%$.

На рисунках 9-11 представлена сравнительная оценка влияния исходных данных на время решения задачи ОГА и методом ветвей и границ. Из рисунка 9 видно, что для больших размерностей (более 150 переменных) время решения задачи ОГА растет не так быстро, как при методе ветвей и границ, в том числе с определением порядка ветвления переменных, что говорит об эффективности и перспективности применения ОГА для получения точного решения задач большой размерности.

Из рисунка 10 видно, что с ростом числа ограничений в решаемой задаче время решения ОГА возрастает по линейному закону и носит менее выраженный характер по сравнению с методом ветвей и границ. Для размерности задач с количеством ограничений меньше 4 время решения задачи методом ветвей и границ с определением порядка ветв-

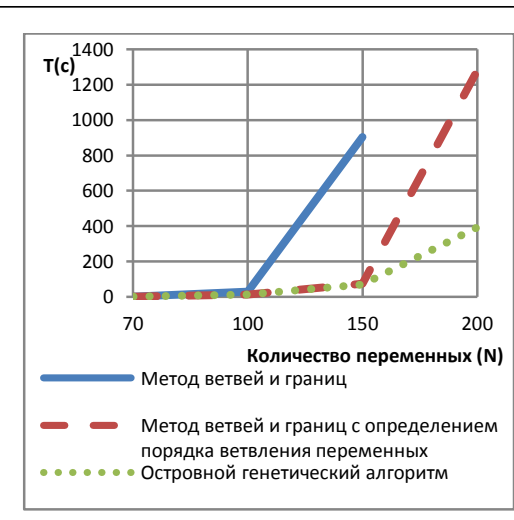

Рис. 9. Зависимость времени решения задач от числа переменных при количестве ограничений $M=4$

Fig. 9. The time for solving a number of variables when the number of restrictions $M=4$

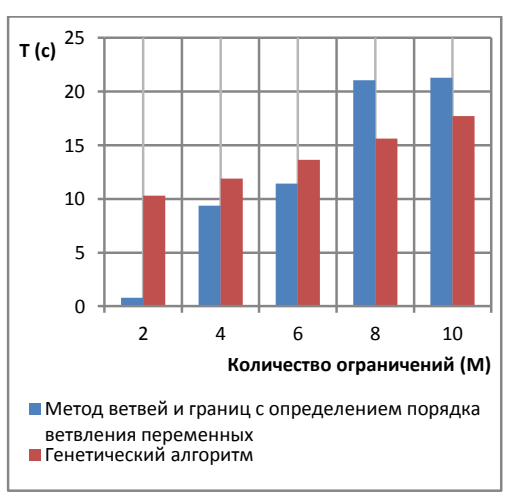

Рис. 10. Влияние числа ограничений на время решения задачи

Fig. 10. The effect of the number of restrictions on the time for solving the problem

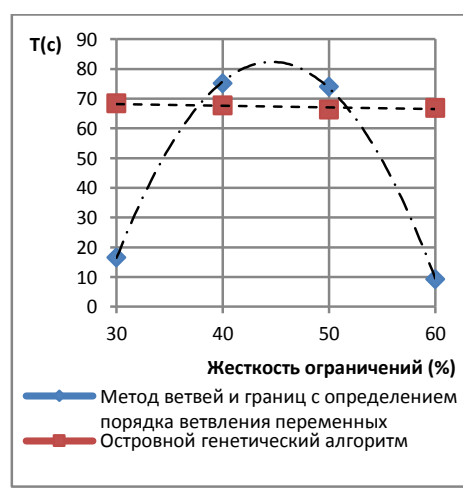

Рис. 11. Зависимость времени решения задачи $150 \times 4$ от жесткости ограничений

Fig. 11. Dependence of $150 \times 4$ problem solving time on constraint rigidity 
Результаты оценки влияния точности решения задач на время решения

Results of assessing the impact of the accuracy of solving problems at the solution time

\begin{tabular}{|c|c|c|c|c|c|c|}
\hline $\begin{array}{c}\text { Размер- } \\
\text { ность } \\
\text { задачи } \\
\end{array}$ & $\begin{array}{c}\text { Время получения } \\
\text { оптимального } \\
\text { решения (сек.) } \\
\end{array}$ & $\begin{array}{c}\text { Оптималь- } \\
\text { ное } \\
\text { решение } \\
\end{array}$ & CКO & $\begin{array}{c}\text { Среднее } \\
\text { значение целевой } \\
\text { функции } \\
\end{array}$ & $\begin{array}{l}\text { Среднее время } \\
\text { решения (сек.) }\end{array}$ & $\begin{array}{c}\text { Относительная } \\
\text { погрешность } \\
\text { решения (\%) } \\
\end{array}$ \\
\hline \multirow{6}{*}{$100 \times 4$} & \multirow{6}{*}{12} & \multirow{6}{*}{2292,61} & 1,87 & 2291,57 & 10,132 & 0,0454 \\
\hline & & & 3,08 & 2290,96 & 7,08 & 0,072 \\
\hline & & & 3,3 & 2288,96 & 4,864 & 0,1593 \\
\hline & & & 4,45 & 2277,34 & 2,218 & 0,6661 \\
\hline & & & 11,39 & 2267,02 & 1,118 & 1,116 \\
\hline & & & 11,79 & 2270,52 & 1,654 & 0,9637 \\
\hline \multirow{5}{*}{$150 \times 4$} & \multirow{5}{*}{69,5} & \multirow{5}{*}{3368,20} & 1,31 & 3367,54 & 37,428 & 0,0195 \\
\hline & & & 3,85 & 3365,56 & 54,524 & 0,0783 \\
\hline & & & 3,03 & 3357,33 & 11,806 & 0,3226 \\
\hline & & & 5,67 & 2933,31 & 3,256 & 12,9117 \\
\hline & & & 9,43 & 2917,90 & 1,642 & 13,369 \\
\hline
\end{tabular}

ления переменных меньше, чем ГА, это объясняется меньшей вычислительной сложностью данного метода для задач малой размерности (с малым числом ограничений).

На рисунке 11 представлена оценка влияния жесткости ограничений в решаемой задаче на время решения задач. Из рисунка видно, что изменение жесткости ограничений не оказывает существенного влияния на время решения задачи ОГА. При этом время решения задачи ОГА оказывается ниже (до $20 \%$ ) времени решения той же задачи методом ветвей и границ с определением порядка ветвления переменных при значении жесткости ограничений в интервале 40-50 \%.
В таблице 6 и на рисунке 12 представлены результаты оценки зависимости влияния точности получаемого решения на общее время решения задачи ОГА. Изменение точности получаемого решения осуществлялось путем варьирования значений параметров ОГА, представленных в таблице 2. Для каждого варианта параметров ОГА задача каждой размерности решалась по 10 раз.

Из таблицы 6 и рисунка 12 видно, что ухудшение качества полученного ОГА решения всего на $0,5 \%$ от оптимального приводит к сокращению времени получения рационального решения в 3-6 раз по сравнению со временем получения оптимального решения. При этом с ростом размерности

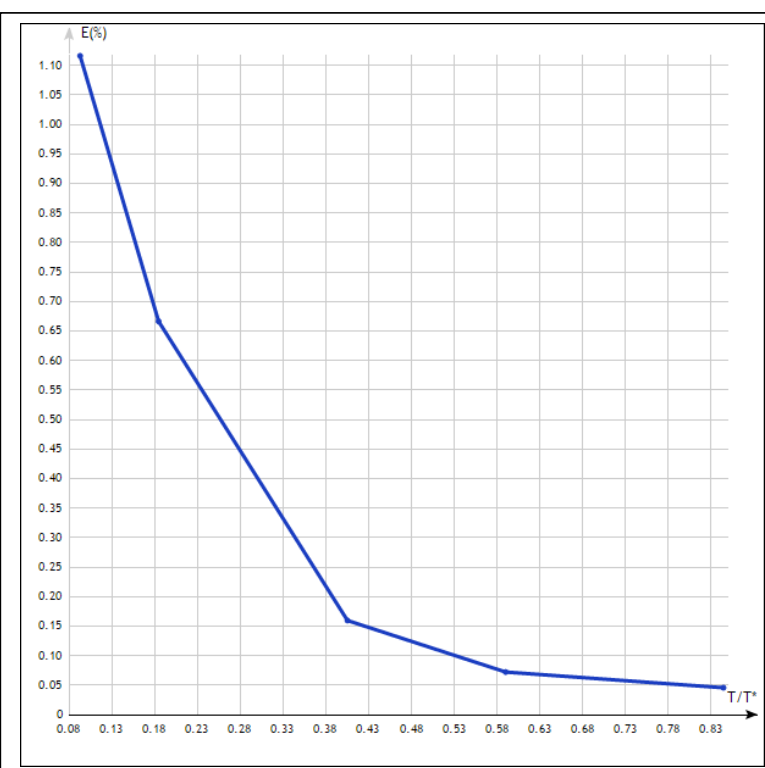

a)

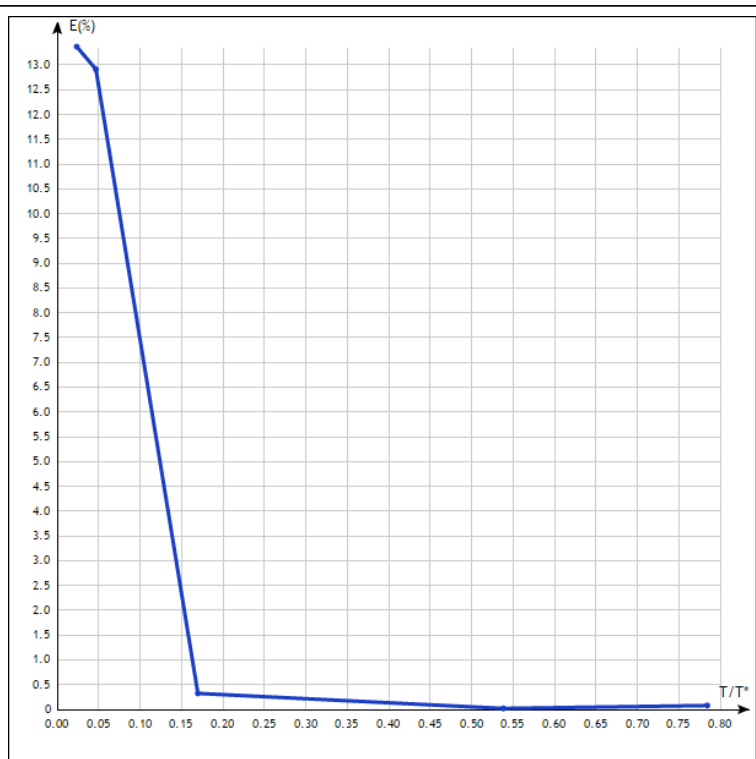

б)

Рис. 12. Оченка влияния точности решения на время решения: а) задача 100×4; б) задача $150 \times 4$ ( $T^{*}$ - время получения оптимального решения)

Fig. 12. Assessment of the impact on the accuracy of the solution and the solution time: a) the task 100×4; б) the task $150 \times 4(T *$ - time optimal solutions) 
решаемой задачи данный эффект становится более явным. Это обстоятельство позволяет сделать вывод о перспективности и целесообразности применения ОГА для оперативного получения рационального (квазиоптимального) решения в условиях жестких временных ограничений.

Таким образом, результаты экспериментальной проверки показали, что применение ОГА для решения задач обеспечения устойчивости функционирования РИС эффективно для задач большой размерности (с числом переменных более 150), задач с жесткостью ограничений в интервале 40-50\%, а также в условиях жестких временных ограничений. В качестве схемы отбора особей целесообразно использовать случайный отбор или адаптивную схему. Использование простого ГА целесообразно для решения задач малой размерности (число переменных до 70). Использование ОГА для получения рационального решения поставленных задач позволит сократить время решения задачи размерности $100 \times 4$ по сравнению с применением точных методов в 5 раз с обеспечением размера относительной ошибки в $0,7 \%$ и в 6-10 раз с обеспечением величины относительной ошибки в $1,5 \%$. С ростом размерности решаемых задач выигрыш по времени получения рационального решения становится еще более существенным.

Метод ветвей и границ целесообразно применять для решения задач обеспечения устойчивости функционирования РИС на этапах их проектирования, когда необходимо получить оптимальное решение и отсутствуют строгие ограничения на время его получения. На этапе эксплуатации, при реконфигурировании системы передачи и обработки данных, в условиях жесткого лимита времени на время реакции системы на отказы и сбои целесообразно применять метод получения рационального (квазиоптимального) решения, основанный на использовании ОГА, который в отличие от существующих приближенных методов обеспечивает за счет обоснованного выбора параметров алгоритма гарантированное получение рационального (квазиоптимального) решения (серии рациональных решений) за время, не превышающее заданное.

\section{Литература}

1. Воронин А.А., Морозов Б.И. Надежность информационных систем. СПб: Изд-во СПбГТУ, 2001. 89 с.

2. Бройдо В.Л. Вычислительные системы, сети и телекоммуникации. 2-е изд. СПб: Питер, 2004. 705 с.

3. Николаев В.И., Брук В.М. Системотехника: методы и приложения. Л.: Машиностроение, 1985. 199 с

4. Есиков Д.О. Обеспечение устойчивого функционирования вычислительных систем за счет обеспечения высоконадежного хранения данных // Фундаментальные и прикладные исследования, разработка и применение высоких технологий в промышленности: сб. стат. XV Междунар. науч.-практич. конф. СПб: Изд-во Политехн. ун-та, 2013. С. 110-118.
5. Повзнер Л.Д. Теория систем управления. М.: Изд-во МГГУ, 2002. $472 \mathrm{c.}$

6. Данилкин Ф.А., Есиков Д.О. Оптимизация распределения информационных ресурсов в вычислительной сети // Инновации в технологиях и образовании: сб. стат. V Междунар. науч. конф. Белово: Изд-во КузГТУ, 2012. Ч. 2. 328 с.

7. Киселев В.Д., Есиков О.В., Кислицын А.С. Теоретические основы оптимизации информационно-вычислительного процесса и состава комплексов средств защиты информации в вычислительных сетях; [под ред. Е.М. Сухарева]. М.: Полиграфсервис XXI век, 2003. 198 с.

8. Есиков Д.О. Задачи обеспечения устойчивости функционирования распределенных информационных систем // Программные продукты и системы. 2015. № 4. С. 133-142.

9. Сергиенко И.В., Гуляницкий Л.Ф., Сиренко С.И. Классификация прикладных методов комбинаторной оптимизации // Кибернетика и системный анализ. 2009. № 5. С. 71-83.

10. Алексеев О.Г. Комплексное применение методов дискретной оптимизации. М.: Наука, 1987. 248 с.

11. Есиков Д.О. Оценка влияния предварительного определения порядка ветвления переменных на эффективность метода ветвей и границ // Междисциплинарные исследования в области математического моделирования и информатики: матер. науч.практич. конф. Ульяновск: SIMJET, 2013. С. 17-21.

12. Бабаев А.А. Организация поиска решений на деревьях детерминированной структуры // Электронное моделирование. 1985. T. 7. № 1. С. 19-25.

13. Есиков Д.О. Повышение эффективности метода ветвей и границ выбором способа организации компьютерных вычислений // Интеллектуальные и информационные системы (Интеллект 2013): сб. стат. Всерос. науч.-практич. конф. Тула: Изд-во ТулГУ, 2014. С. 143-147.

14. Yesikov D.O. Increase of level of stability of functioning of systems of storage and data processing at the expense of realization of actions for ensuring safety of information. Proc. 3rd Intern. Conf. High Performance Computing (HPC-UA 2013). Ukraine, Kiev, 2013, pp. 416-420.

15. Есиков О.В., Есиков Д.О. Математические модели и алгоритмы обеспечения сохранности информации // Изв. Тульского гос. ун-та: Технические науки. 2013. Вып. 9. Ч. II. C. $110-118$.

16. Есиков Д.О., Ивутин А.Н. Программа решения задач целочисленного линейного программирования с булевыми переменными. Свид. о гос. регистр. прогр. для ЭВМ № 2014619322. Госрегистр. в Реестре прогр. для ЭВМ 15.09.2014.

17. Рутковская Д., Пилиньский М., Рутковский Л. Нейронные сети, генетические алгоритмы и нечеткие системы; [пер. с пол. И.Д. Рудинского]. М.: Горячая линия-Телеком, 2004. 383 с.

18. Holland J.H. Adaptation in Natural and Artificial Systems: 2nd ed. Cambridge, MA: MIT Press, 1992.

19. Гладков Л.А., Курейчик В.В., Курейчик В.М. Генетические алгоритмы. М.: Физматлит, 2006. 320 с.

20. Нгуен Минь Ханг. Применение генетического алгоритма для задачи нахождения покрытия множества // Труды ИСА РАН. 2008. № 33. С. 206-219.

21. Есиков Д.О., Ивутин А.Н., Ларкин Е.В. Программа решения задач целочисленного программирования с булевыми переменными островным генетическим алгоритмом. Свид. о гос. регистр. прогр. для ЭВМ № 2015663468. Госрегистр. в Реестре прогр. для ЭВМ 18.12.2015.

22. Yesikov D.O., Ivutin A.N. Rational values of parameters of island genetic algorithms for the effective solution of problems of ensuring stability of functioning of the distributed information systems. Proc. 5th Mediterranean Conf. on Embedded Computing (MECO) 2016, pp. 309-312. DOI: 10.1109/MECO.2016.7525769.

23. Карпенко А.П. Популяционные алгоритмы глобальной поисковой оптимизации. Обзор новых и малоизвестных алгоритмов // Информационные технологии. 2012. № 7. С. 13-15. 


\section{EVALUATING THE EFFECTIVENESS OF SUSTAINABILITY PROBLEM SOLVING METHODS OF DISTRIBUTED INFORMATION SYSTEM FUNCTIONING}

D.O. Esikov ${ }^{1}$, Postgraduate Student, mcgeen4@gmail.com

${ }^{1}$ Tula State University, Lenina Ave. 92, Tula, 300012, Russian Federation

Abstract. To make informed decisions regarding the organization of data storage and processing to ensure the sustainability of distributed information systems the paper proposes to use a complex of mathematical models for optimizing the distribution of functional task software elements over network nodes; for optimizing the distribution of information resources by data storage and processing centers; for optimizing technical means of data storage and processing system; for optimizing the allocation of resources by information storage and processing centers. It is shown that these problems are related to the class of optimization problems with discrete Boolean variables.

The paper proposes and experimentally verifies the branch-and-bound method and the method of genetic algorithms to solve formalized problems. In order to improve the efficiency of the branch-and-bound method the paper proposes preliminary determination of the variable branching order using an approximation method for solving the dual problem once. The article shows an experimental verification of the effectiveness of the branch-and-bound method for solving problems of ensuring distributed information system sustainability including the use of the algorithm of predetermined order of branching variables. The author estimates the initial data influence on overall performance of the branch-and-bound method. The paper shows the most effective strategies of variables branching to solve the designed tasks. There are variations of major operators, as well as initialization circuits of a genetic algorithm initial population to solve the problems of ensuring distributed information system sustainability.

To improve the quality of the solution, which is obtained using a genetic algorithm, the author justifies the use of an adaptive scheme of individuals' reproduction and an computational island circuit. He also experimentally checks the effectiveness of the proposed genetic and island genetic algorithms. The paper determines the parameters of genetic algorithms for ensuring maximum quality of the solutions and proves ability to manage the accuracy of the obtained solution by changing the algorithm parameters when introducing time restrictions for solving. There is a comparative assessment of the branch-andbound method and the island genetic algorithm for solving formal problems. The paper also shows areas of their effective application.

Keywords: distributed information system, operation stability, mathematical models, branch-and-bound method, dual problem, genetic algorithm, island genetic algorithm, adaptive reproduction scheme.

\section{References}

1. Voronin A.A., Morozov B.I. Nadezhnost informatsionnykh sistem [Information System Reliability]. St. Petersburg, SPbPU Publ., 2001, 89 p.

2. Broido V.L. Vychislitelnye sistemy, seti i telekommunikatsii [Computer Systems, Networks and Telecommunications]. Piter Publ., 2nd ed., 2004, 705 p.

3. Nikolaev V.I., Brooke V.M. Sistemotekhnika: metody i prilozheniya [System engineering: methods and applications]. L., Mashinostroenie Publ., 1985, 199 p.

4. Yesikov D.O. Sustainable operation of computer systems by providing highly reliable data storage. Fundamentalnye $i$ prikladnye issledovaniya, razrabotka i primenenie vysokikh tekhnology v promyshlennosti: sb. stat. XV Mezhdunar. nauch.praktich. konf. [Proc. 15 Int. Science and Practice Conf. "Fundamental and Applied Research, Development and Application of High Technologies in the Industry”]. St. Petersburg, Polytechnic Univ. Publ., 2013, pp. 110-118 (in Russ.).

5. Povzner L.D. Teoriya sistem upravleniya [Theory of Control Systems]. Moscow, MSMU Publ., 2002, $472 \mathrm{p}$.

6. Danilkin F.A., Yesikov D.O. Optimization of informational resources distribution in a computer network. Innovatsii v tekhnologiyakh i obrazovanii: sb. stat. V Mezhdunar. nauch. konf. [Proc. 5th Int. Scientific Conf. "Innovations in Technologies and Education"]. KuzGTU branch in Belovo Publ., 2012, part 2, 328 p.

7. Kiselev V.D., Esikov O.V., Kislitsyn A.S. Teoreticheskie osnovy optimizatsii informatsionno-vychislitelnogo protsessa $i$ sostava kompleksov sredstv zashchity informatsii v vychislitelnykh setyakh [Theoretical bases of information and computing process optimization and structure of information security systems in computer networks]. E.M. Sukharev (Ed.). Moscow, Poligrafservis XXI vek Publ., 2003, 198 p.

8. Yesikov D.O. The problems of ensuring distributed information systems sustainability. Programmnye produkty $i$ sistemy [Software and Systems]. 2015, no. 4 (112), pp. 133-142 (in Russ.).

9. Sergienko I.V., Gulyanitsky L.F., Sirenko S.I. Classification of combinatorial optimization applied methods. Kibernetika i sistemny analiz [Cybernetics and Systems Analysis]. 2009, no. 5, pp. 71-83.

10. Alekseev O.G. Kompleksnoe primenenie metodov diskretnoy optimizatsii [Complex Application of Discrete Optimization Methods]. Moscow, Nauka Publ., 1987, 248 p.

11. Yesikov D.O. Assessing the impact of pre-determining the order of the branching variables on the efficiency of branch and bound method. Mezhdistsiplinarnye issledovaniya $v$ oblasti matematicheskogo modelirovaniya i informatiki: mater. nauch.-praktich. konf. [Proc. Science and Practice Conf. "Interdisciplinary research in the field of mathematical modeling and computer science"]. Ulyanovsk, SIMJET Publ., 2013, pp. 17-21 (in Russ.).

12. Babaev A.A. Organization of Decision Search on Deterministic Structure Trees. Elektronnoe modelirovanie [Electronic modeling]. 1985, vol. 7, no. 1, pp. 19-25 (in Russ.) 
13. Yesikov D.O. Improving the efficiency of branch-and-bound method selection mode of organization of computing. Intellektualnye i informatsionnye sistemy (Intellekt 2013): sb. stat. Vseros. nauch.-praktich. konf. [Proc. All-Russian Science and Practice Conf. "Intellectual and information systems. Intellect 2013"]. Tula, Tula State Univ. Publ., 2014, pp. 143-147 (in Russ.).

14. Yesikov D.O. Increase of level of stability of functioning of systems of storage and data processing at the expense of realization of actions for ensuring safety of information. Proc. 3rd Int. Conf. "High Performance Computing" (HPC-UA 2013). Ukraine, Kyiv, 2013, pp. 416-420.

15. Yesikov O.V., Yesikov D.O. Mathematical models and algorithms to ensure data safety. Izv. Tulskogo gos. un-ta: Tekhnicheskie nauki [News of the Tula State Univ. Engineering Sciences]. 2013, iss. 9, part 2, Tula, pp. 109-118 (in Russ.).

16. Esikov D.O., Ivutin A.N. Programma resheniya zadach tselochislennogo lineynogo programmirovaniya s bulevymi peremennymi [A Problem-solver for integer linear programming problems with Boolean variables]. State registration Certificate of the computer program № 2014619322. 2014 (in Russ.).

17. Rutkowski J., Pilinsky M., Rutkowski L. Neural networks, genetic algorithms and fuzzy systems. (Russ.ed.: I.D. Rudinsky. Moscow, Goryachaya Liniya-Telecom Publ., 2004, 383 p.).

18. Holland J.H. Adaptation in Natural and Artificial Systems. 2nd ed. Cambridge, MA, MIT Press, 1992.

19. Gladkov L.A., Kureychik V.V., Kureychik V.M. Geneticheskie algoritmy [Genetic algorithms]. Moscow, Fizmatlit Publ., 2006, 320 p.

20. Nguyen M.H. Application of a genetic algorithm to the set coating finding problem. Trudy ISA RAN [Proc. ISA RAS]. 2008, no. 33, pp. 206-219 (in Russ.).

21. Esikov D.O., Ivutin A.N., Larkin E.V. Programma resheniya zadach tselochislennogo programmirovaniya s bulevymi peremennymi ostrovnym geneticheskim algoritmom [A Program for Solving Integer Programming Problems with Boolean Variables Using an Island Genetic Algorithm]. State registration Certificate of the computer program № 2015663468. 2015 (in Russ.).

22. Yesikov D.O., Ivutin A.N. Rational values of parameters of island genetic algorithms for the effective solution of problems of ensuring stability of functioning of the distributed information systems. Proc. 5th Mediterranean Conf. on Embedded Computing (MECO). 2016, pp. 309-312.

23. Karpenko A.P. Population algorithms of global search optimization. Overview of new and little-known algorithms. Informatsionnye tekhnologii [Information Technologies]. 2012, no. 7, pp. 13-15 (in Russ.).

\section{Примеры бибциографического описания статьи}

1. Есиков Д.О. Оценка эффективности методов решения задач обеспечения устойчивости функционирования распределенных информационных систем // Программные продукты и системы. 2017. Т. 30. № 2. C. 241-256; DOI: 10.15827/0236-235X.118.241-256.

2. Esikov D.O. Evaluating the effectiveness of sustainability problem solving methods of distributed information system functioning. Programmnye produkty $i$ sistemy [Software \& Systems]. 2017, vol. 30, no. 2, pp. 241-256 (in Russ.); DOI: 10.15827/0236-235X.118.241-256. 